\title{
Radiotherapy for isolated occult subfoveal neovascularisation in age related macular degeneration: a pilot study
}

Guy Donati, Dominique Soubrane, Maddalena Quaranta, Gabriel Coscas, Gisele Soubrane

\begin{abstract}
Backgroundlaims-Teletherapy has been proposed as a possible treatment for choroidal neovascular membranes (CNV), secondary to age related macular degeneration (AMD) not amenable to laser photocoagulation. The aim of this prospective study has been to investigate the effect of teletherapy on isolated occult choroidal neovascular membranes of subfoveal location.
\end{abstract}

Methods-28 AMD patients presenting with retrofoveal isolated occult CNV demonstrated by fluorescein angiography were treated by external beam radiation. A complete ophthalmological examination, fluorescein angiography, and indocyanine green angiography (ICG) were performed within 15 days before treatment and repeated at follow up. A total dose of $16 \mathrm{~Gy}$ was applied in four sessions of $4 \mathrm{~Gy}$ using a $4 \mathrm{MeV}$ photon beam. Follow up ranged from 6 to 9 months (mean follow up 6.4 months).

Results-Visual acuity was found to be visual acuity was of 3-6 lines in $18 \%$ and of more than 6 lines in $10 \%$ of the eyes at last examination. On fluorescein angiography the size of the lesion area was found to be stable in $67 \%$, decreased in $13 \%$, and increased in $20 \%$ of the cases. On ICG angiography the size of the CNV was stable in $93 \%$ and increased in $7 \%$ of the cases. All the eyes experiencing a visual acuity decrease showed either no change or an increase in size of the membrane on fluorescein angiography and/or on ICG.

Department of Clinical Neurosciences,

University Hospitals of

Geneva

G Donati

University Eye Clinic, Hospital of Creteil, University of Paris XII, Paris, France

D Soubrane

M Quaranta

G Coscas

G Soubrane

Correspondence to: G Soubrane, University Eye Clinic, Centre Hospitalier Intercommunal, 40 Avenue de Verdun, 94010 Créteil, France

Accepted for publication 3 December 1998 subfoveal CNV.

(Br f Ophthalmol 1999;83:646-651) the major cause of legal blindness in the developed countries in people aged 50 years or more. ${ }^{12}$ The prevalence of AMD increases with aging to a prevalence of about $30 \%$ in people older than 75 years. ${ }^{23}$ As it is estimated that the section of the population over the age of 85 will be five million by the year 2000 in the USA and tripled by the middle of the next century, it is expected that AMD will be an even greater health problem in the future. ${ }^{4}$ The most stable in $68 \%$ of the cases. The decrease in

severe visual loss occurs in patients with AMD complicated by neovascular membranes. ${ }^{13}$ Such patients account for $12 \%$ of AMD, but for $88 \%$ of legal blindness cases. ${ }^{1}$

Neovascular membranes complicating AMD are subdivided into well defined and occult on fluorescein angiography. Well defined choroidal neovascular membranes (CNV) are characterised by a well demarcated area of early hyperfluorescence with progressive leakage in the late phase. ${ }^{5}$ Occult CNV lack the typical features of well defined CNV. They show either areas of early hyperfluorescence (that are neither as discrete nor as bright as areas of well defined CNV) followed by stippled hyperfluorescence with staining of the fibrovascular tissue and leakage within the neurosensory retinal space during the late phase, or late phase fluorescein leakage of an undetermined source, which appears as speckled hyperfluorescence with pooling of dye in the overlying subsensory retinal space. ${ }^{5-7}$

Differentiating well defined from occult $\mathrm{CNV}$ is of fundamental clinical significance. At present laser photocoagulation remains the only worldwide demonstrated treatment for well defined neovascular membranes (of extrafoveal, juxtafoveal, and subfoveal location $)^{58-15}$ complicating AMD. However, laser photocoagulation failed to demonstrate any significant benefit in eyes with occult $\mathrm{CNV}^{7}{ }^{16-18}$ As it is estimated that $60-70 \%$ of the CNV in AMD are occult at the time of diagnosis, ${ }^{19}{ }^{20}$ there is strong need for new therapies. Radiotherapy is a treatment method that has been recently proposed to treat subfoveal CNV in AMD. Pilot studies showed a beneficial effect in well defined or partially well defined CNV, ${ }^{21-26}$ but only scarce evidence is available on its effect on occult CNV alone. ${ }^{26}$ We present here the first results of a pilot study conducted to investigate the effect of radiotherapy on isolated occult $\mathrm{CNV}$ of subfoveal location before initiating a large randomised trial.

Age related macular degeneration (AMD) is

\section{Patients and methods}

PATIENT SELECTION

Twenty eight consecutive patients with isolated occult subfoveal CNV, referred to the University Eye Clinic of Créteil, were included in this study. The eligibility criteria are summarised in Table 1. Briefly, all patients were over the age of 50 and had evidence of AMD. Fluorescein angiography showed isolated subfoveal occult CNV, thus making the patients ineligible for laser photocoagulation. The patients were 


\section{Table 1 Inclusion criteria}

Age $>50$ years

Diagnosis of age related macular degeneration including drusen, pigment epithelial atrophy or migration in the fellow eye, and/or pigment epithelial detachment in the study, and/or fellow eye

Symptoms of decreased visual acuity within the past 3 months, and/or retinal haemorrhages, and/or serous posterior epithelial detachment in the study eye

Angiographic evidence of subfoveal occult neovascularisation, with or without serous pigment epithelial detachment, without detectable fibrous tissue in the study eye

Initial visual acuity ranging from $20 / 25$ to $20 / 200$ in the study eye

No prior photocoagulation in the study eye

No other ocular disease that could significantly affect visual acuity in the study eye

Table 2 Baseline characteristics by initial examination

\begin{tabular}{ll}
\hline Characteristics & No (\%) of patients \\
\hline Age (years): & \\
$60-69$ & $4(14)$ \\
$70-79$ & $19(68)$ \\
$80+$ & $5(18)$ \\
Sex: & $15(54)$ \\
Female & $13(46)$ \\
Male & \\
Features associated with occult & \\
CNV: & $28(100)$ \\
Neurosensory retinal detachment & $16(57)$ \\
Blood & $3(11)$ \\
Serous PED & $7(25)$ \\
Visual acuity: & $10(36)$ \\
$20 / 25-20 / 40$ & $7(25)$ \\
$20 / 50-20 / 80$ & $4(14)$ \\
$20 / 100-20 / 160$ & $2(7)$ \\
２0/200 & $10(36)$ \\
Area of lesion on fluorescein angiography: & \\
$<1$ DA & $9(32)$ \\
$>1$ to $<3.5$ DA & $6(21)$ \\
$>3.5$ to $<6$ DA & $1(4)$ \\
$>6$ to $<9$ DA & \\
$>9$ DA & $3(20)$ \\
Area of lesion on ICG angiography: & $6(40)$ \\
$<1$ DA & $4(27)$ \\
$>1$ to $<3.5$ DA & $2(13)$ \\
$>3.5$ to $<6$ DA & 0 \\
$>6$ to $<9$ DA & \\
$>9$ DA & \\
\hline
\end{tabular}

$\mathrm{CNV}=$ choroidal neovascularisation; $\mathrm{PED}=$ pigment epithelium detachment; $\mathrm{DA}=$ disc area; $\mathrm{ICG}=$ indocyanine green angiography.

informed of the nature of their condition, as well as of the experimental nature and the potential complications of ocular radiotherapy. All the patients agreed to participate in the study.

BASELINE AND FOLLOW UP EVALUATION

All recruited patients were subjected to an ophthalmic examination including best corrected distance visual acuity, biomicroscopic fundus examination, and fluorescein angiography. Indocyanine green angiography (ICG) was additionally obtained in 15 cases. Examinations were performed within 15 days before the beginning of radiotherapy and repeated every 3 months during follow up. Follow up ranged from 6 to 9 months for all patients. Changes in visual acuity from baseline examination were graded as mild (decrease or increase of 3-6 lines) and severe (decrease or increase of more than 6 lines). The size of each lesion on the fluorescein angiogram was determined by superimposing a transparent sheet with circles of 1-12 disc areas determined from each patient's red free photograph. A difference in size of at least 0.5 disc area was considered significant. To avoid bias in evaluation, baseline and follow up photographs were assessed in a masked fashion by one of the authors.

\section{STATISTICAL ANALYSIS}

Comparison of subgroups with respect to lesion size on fluorescein and ICG angiography and changes in visual acuity were assessed with the Student's test (independent $t$ test). The significance level was chosen at $\mathrm{p}=0.05$.

TREATMENT PROTOCOL

Teletherapy was carried out using a technique similar to that previously described by others. ${ }^{21} 22{ }^{25}$ Briefly, a $4 \mathrm{MeV}$ photons beam was used to deliver a total dose of $16 \mathrm{~Gy}$ in four sessions of $4 \mathrm{~Gy}$. The total dose $(100 \%)$ was administered to an area of $1 \mathrm{~cm}^{2}$ in the macular region; outside this area the dose decreased progressively to $50 \%$ at the posterior pole of the lens. The lateral field beam was tilted at $15^{\circ}$ in order to reduce irradiation to the contralateral eye (less than $10 \%$ of the total dose).

\section{Results}

BASELINE EXAMINATION

Baseline characteristics are summarised in Table 2. Most of the patients were between 70 and 79 years of age, $54 \%$ of them being males and $46 \%$ females. Distribution of initial visual acuity ranged from $20 / 25$ to $20 / 200,25 \%$ with $20 / 40$ or better, $36 \%$ between $20 / 50$ to $20 / 80$, $25 \%$ between $20 / 100$ to $20 / 150$, and $14 \%$ with 20/200. Biomicroscopic examination showed neurosensory retinal detachment in all eyes, intraretinal and/or subretinal haemorrhages were present in $57 \%$ of the eyes and serous pigment epithelial detachment (PED) in 11\% of the cases. Fluorescein angiograms showed occult CNV involving the centre of the fovea in all cases. In addition, ICG showed a late staining hyperfluorescent plaque involving the centre of the fovea in all cases.

\section{CHANGES IN VISUAL ACUITY FROM BASELINE} EXAMINATION

Change in visual acuity from baseline are summarised in Table 3: $72 \%$ of the eyes had no decrease or even an increase in visual acuity, $18 \%$ experienced a moderate (between 3 and 6 lines), and 10\% a severe (more than 6 lines) decrease in visual acuity.

Table 3 Changes in visual acuity by lesion characteristics at baseline examination

\begin{tabular}{|c|c|c|c|c|c|c|}
\hline & \multirow[b]{2}{*}{ No of eyes } & \multicolumn{5}{|c|}{ Visual acuity change at follow up (\% of eyes) } \\
\hline & & $\begin{array}{l}\text { worse } \\
<-6\end{array}$ & $\begin{array}{l}\text { worse } \\
<-3 \text { to }-6\end{array}$ & $\begin{array}{l}\text { same } \\
>-2 \text { to }<+2\end{array}$ & $\begin{array}{l}\text { improved } \\
>+3 \text { to }+6\end{array}$ & $\begin{array}{l}\text { improved } \\
>+6\end{array}$ \\
\hline Occult CNV & 28 & 10 & 18 & 68 & 0 & 4 \\
\hline Occult with blood or PED & 16 & 19 & 13 & 68 & 0 & 0 \\
\hline Occult, no blood, no PED & 12 & 0 & 25 & 74 & 0 & 1 \\
\hline
\end{tabular}


Table 4 Changes in lesion area at follow up on fluorescein angiography and ICG

\begin{tabular}{lllll}
\hline & & \multicolumn{2}{l}{$\begin{array}{l}\text { \% of eyes by change in size of lesion area at } \\
\text { follow up }\end{array}$} \\
\cline { 3 - 5 } & No of eyes & $\begin{array}{l}\text { Increased by at } \\
\text { least } 0.5 \text { DA }\end{array}$ & $\begin{array}{c}\text { Same } \\
\text { Decreased by at } \\
\text { least 0.5 DA }\end{array}$ \\
\hline FA: & 28 & 20 & 67 & 13 \\
Occult CNV & 16 & 19 & 62 & 19 \\
Occult with blood or PED & 12 & 0 & 92 & 8 \\
Occult with no blood, no PED & 15 & 7 & 93 & 0 \\
ICG: & 10 & 0 & 100 & 0 \\
Occult CNV & 5 & 20 & 60 & 20 \\
Occult with blood or PED & & & \\
Occult with no blood, no PED & 5 & & & \\
\hline
\end{tabular}

$\mathrm{FA}=$ fluorescein angiography; $\mathrm{ICG}=$ indocyanine green angiography; $\mathrm{DA}=$ disc areas; $\mathrm{CNV}=$ choroidal neovascularisation; $\mathrm{PED}=$ pigment epithelium detachment.

RELATION BETWEEN MORPHOLOGY AND CHANGES IN VISUAL ACUITY

The changes in visual acuity in relation to morphological features at baseline are summarised in Table 3. The groups of eyes presenting with blood or serous PED at baseline had a significantly poorer outcome than those without: five $(34 \%)$ eyes suffered a significant decrease in visual acuity, versus three $(25 \%)$ in the group presenting without blood or serous PED. Statistical analysis of the visual acuity changes confirmed a significative difference between the outcome in the two groups (mean initial visual acuity in the group presenting with blood and/or serous PED at baseline 0.3 (SD
$0.1)$, mean final VA $0.2(0.1), p=0.036$, mean initial VA in the group presenting without it 0.4 $(0.3)$, mean final VA $0.4(0.2), \mathrm{p}=0.77)$.

CHANGES IN LESION SIZE

The changes in size at follow up compared with baseline measured on fluorescein and ICG are summarised in Table 4: 19 (67\%) eyes showed either no change or even a slight decrease in size of the lesion area on fluorescein angiography (Figs 1 and 2). Statistical analysis showed no significant difference between initial and final size of the lesion area (mean size of disc areas initially 4.2 (SD 2.6), $v$ finally 4.6 (2.8), $\mathrm{p}=0.88$ ). Moreover, in the group of patients in which ICG was obtained the size of the hyperfluorescent plaque was stable in 14 (93\%) eyes. Statistical analysis confirmed the absence of any significant difference between initial and final size of the lesion area (mean size in disc areas initially 3.8 (2.6), $v$ finally $3.8(2.4), p=1)$. Comparison of the size of the lesion obtained on ICG and fluorescein angiography (mean size in disc areas initially 4.8 (2.7), finally 5.1 (3.1)) showed no significant difference $(p=0.25$ for the initial size, $\mathrm{p}=0.17$ for the final size). The size of the lesion area on ICG was the same or less than the one measured on fluorescein angiography in all cases both at baseline and at follow up. A decrease or increase in the size of the lesion
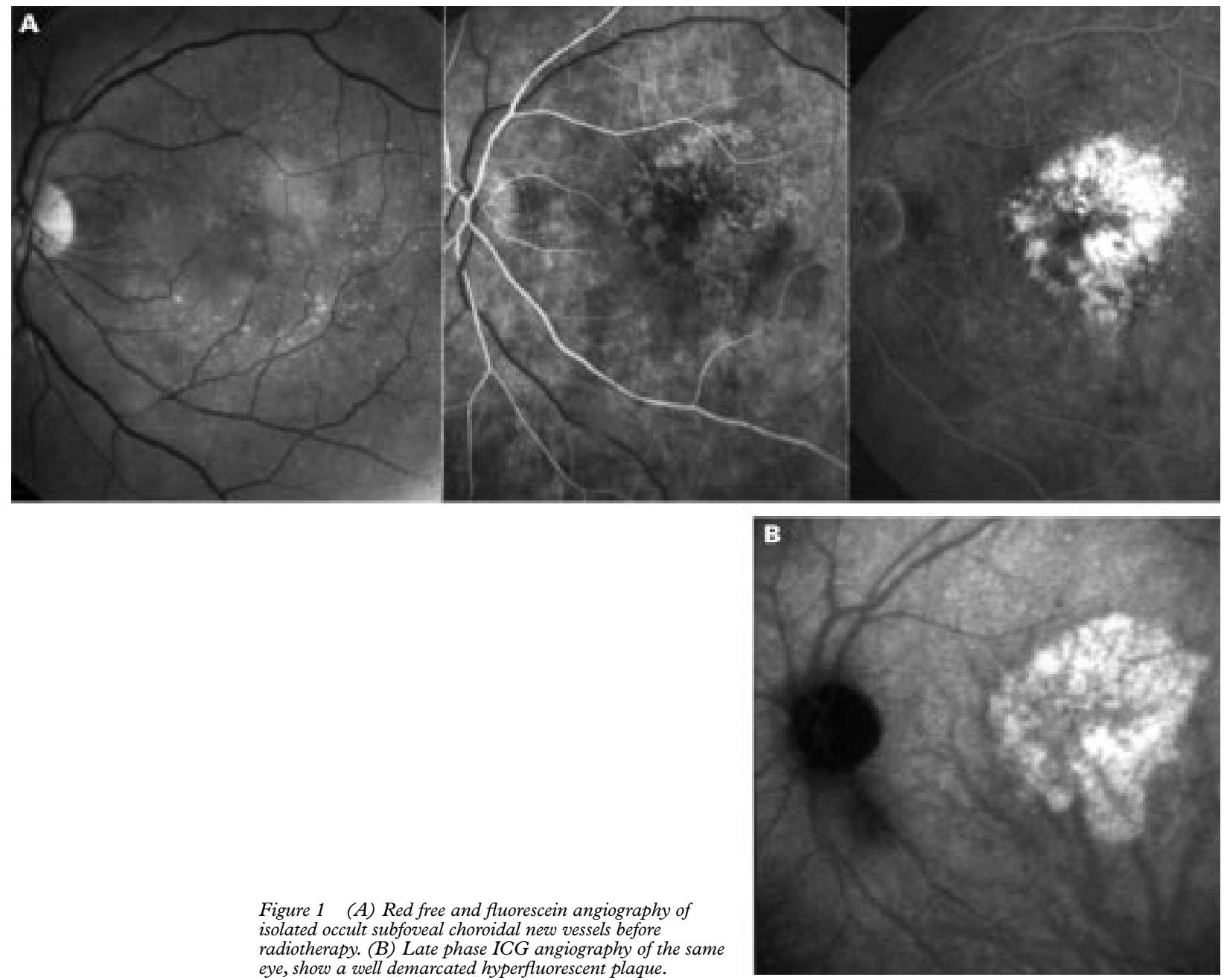

Figure 1 (A) Red free and fluorescein angiography of radiotherapy. $(B)$ Late phase ICG angiography of the same eye, show a well demarcated hyperfluorescent plaque. 


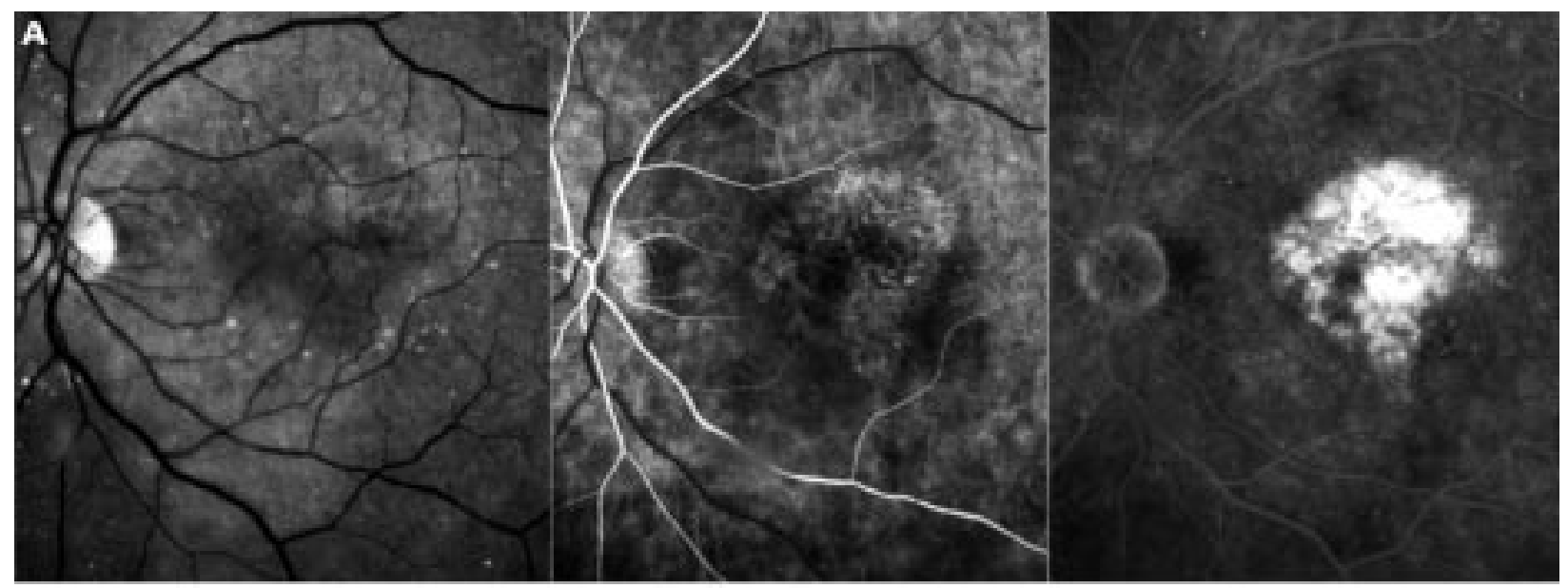

Figure 2 Red free picture, fluorescein, and ICG angiography 9 months after radiotherapy. Note as, on fluorescein angiography, the zone occupied by the choroidal new vessels seems the same as before treatment. (B) On ICG angiography, the late stainining plaque is slightly more extended than before treatment.

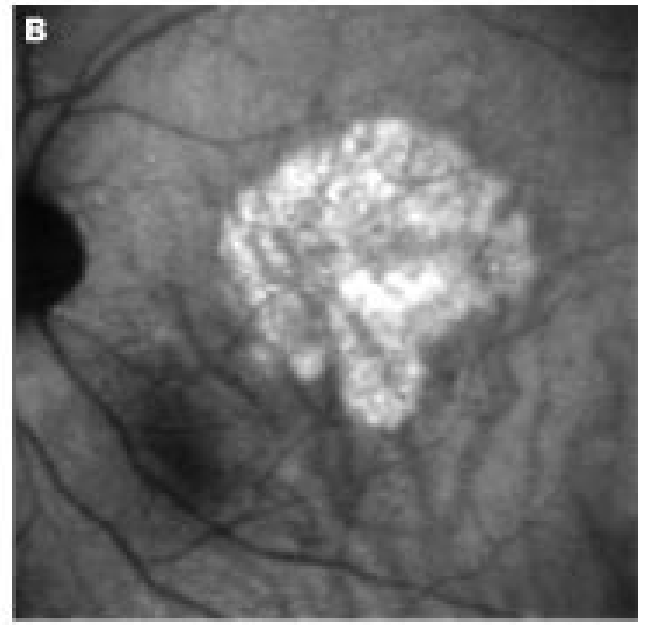

area on fluorescein angiography was not significantly correlated with changes in the size of the late staining plaque on ICG.

CHANGES IN LESION CHARACTERISTICS

Blood and/or serous PED observed at baseline in $19(68 \%$ ) eyes (see Table 1 ) regressed in all cases but one at follow up. Of the nine eyes that presented without blood or serous PED at baseline two (22\%) had significant bleeding during follow up, which was still present at final examination. Some neuroretinal detachment was present in all cases at baseline (see Table 1). It persisted in $23(82 \%)$ eyes at the follow up on biomicroscopic examination. Fluorescein angiography showed persistent leakage of dye to some extent in all eyes (Fig 2 A); however, at final follow up examination a significant degree of chorioretinal atrophy was observed in four (15\%) of the eyes which corresponded to a significant decrease in the lesion area on fluorescein angiography. Development of some fibrous tissue occurred in only one of the eyes presenting with either blood or serous PED at baseline examination.

RELATION BETWEEN LESION CHARACTERISTICS AND VISUAL ACUITY

Visual acuity was found to be stable in 50\% of the eyes showing no changes in size of lesion area on fluorescein angiography and/or on ICG. No significant decrease in visual acuity was observed in the eyes showing a decrease in

size of lesion area on fluorescein angiography. Finally, all the eyes suffering a decrease in visual acuity at follow up examination showed either no change or an increase in size of lesion area on fluorescein angiography and/or on ICG.

\section{Discussion}

Studies on the natural history of eyes with subfoveal occult neovascular membranes have shown that long term visual outcome is poor. ${ }^{671827}$ At present there is no treatment of proved value for occult subfoveal CNV. Confluent or scatter laser photocoagulation was shown to be of no benefit in occult subfoveal CNV. ${ }^{716}{ }^{17}$ Perifoveal laser photocoagulation has been shown to preserve visual acuity in the short term in occult subfoveal CNV, but the long term visual outcome was still poor. Among experimental therapies, interferon alfa- $2^{28}$ was shown to be of no benefit in the treatment of CNV in AMD. Surgical excision only addresses well defined subfoveal $\mathrm{CNV}^{29-32}$ and photodynamic therapy is currently under investigation. Radiotherapy is another alternative treatment for subfoveal CNV in AMD. Since the original publication of Chakravarthy et $a l,{ }^{21}$ there has been increasing interest in this treatment as it is noninvasive and is reported to have no systemic or local side effects at the low doses used. ${ }^{21-23} 2526$ Most of the current studies report concerned 
well defined subfoveal $\mathrm{CNV}^{21-25} 3334$ Only scarce and controversial results are available on the effect of radiotherapy on a well selected group of eyes with occult subfoveal CNV. ${ }^{26}{ }^{35}$

ANATOMICAL RESULTS

According to our data, external beam radiotherapy seems to have a stabilising effect on the growth of subfoveal occult $\mathrm{CNV}$ as judged by the observed modifications in lesion size. Firstly, most of the eyes ( $80 \%$ ) showed either a decrease or no change in size during follow up on fluorescein angiography. Eyes presenting without blood or serous PED at baseline did significantly better, $100 \%$ showing either no change or a decrease in size. This result differs from the reports on natural history of occult $\mathrm{CNV}$ that showed an increase in size in all the cases after at least 6 months of follow up. ${ }^{60}$ Secondly, significant fibrovascular proliferation was observed at follow up in only one eye that presented with blood at baseline, an event that was previously reported to occur after at least 6 months of observation in $22 \%$ of the eyes presenting with the same characteristics at baseline. ${ }^{20}$ Thirdly, no case of growth of well defined CNV was observed, a complication previously reported to occur in as many as $50 \%$ of the eyes during natural history of occult CNV after 9-12 months of follow up. ${ }^{70}$ Finally, the onset of an atrophic scar was observed in $15 \%$ of the cases. These results are in accordance with previous reports on the evolution of well defined subfoveal CNV after radiotherapy. ${ }^{24}$ Occult $\mathrm{CNV}$, as well defined $\mathrm{CNV}$, react to external beam irradiation with a reduction of the trend of growth and of disciform scar formation. ${ }^{36}$

\section{FUNCTIONAL RESULTS}

In the present study $72 \%$ of the eyes had stable or increased vision at follow up examination, $18 \%$ suffered from a mild decrease, and $10 \%$ from a severe decrease in visual acuity. Indeed, the natural course of such lesions has been shown to be deleterious for the visual function with a longer follow up as only $37 \%$ of eyes have been shown to retain a stable visual acuity, $21 \%$ suffering a mild and $42 \%$ a severe decrease in visual acuity after at least 6 months of follow up. ${ }^{720}$ However, final visual acuity was slightly worse $(68 \%$ of eyes showing no significant change in visual acuity) than previously reported by others. ${ }^{26}$ As inactive and self limiting CNV have been reported to occur relatively frequently among occult lesions, ${ }^{37}$ this could be due to the fact that some of the lesion included in previous studies were inactive and self limiting choroidal CNV. Identification of the prognostic factors for functional visual outcome in eyes presenting with occult CNV is therefore necessary. The presence of blood was recently suspected as being such a factor, but could not be demonstrated. ${ }^{20}$ In our study, eyes presenting with blood and/or serous PED at baseline had a significantly worse functional outcome at follow up than eyes without blood and/or serous PED. Therefore, the results of the present study show that external beam irradiation has a beneficial effect on the visual acuity of eyes with occult subfoveal CNV, presenting with a spontaneous unfavourable aspect at baseline. Moreover, it suggests that occult subfoveal CNV, presenting with blood or serous PED on biomicroscopic examination may constitute a subgroup with a particularly bad prognosis. Such cases had a significantly less favourable evolution after radiotherapy than eyes presenting with only a decrease in visual acuity and a neuroretinal detachment at baseline.

RELEVANCE OF ICG IN THE EVALUATION OF OCCULT CNV

ICG has recently been shown to allow visualisation of CNV being defined as occult on fluorescein angiography, ${ }^{38-40}$ ICG allowing a more precise delineation of the lesion. In our study, the size of the lesion was similar on ICG and on fluorescein angiography at baseline and at follow up, and $93 \%$ of the eyes showing no changes in lesion size at follow up. However, changes in size on fluorescein angiography could not be correlated with modifications in size on ICG. As hyperfluorescent plaques on ICG are thought to represent active $\mathrm{CNV},{ }^{39}$ this indicates that changes in fluorescein angiography, and particularly changes in the size of the leakage area, may occur without modifications in the size of the active CNV, therefore suggesting that ICG angiography could be more valuable than fluorescein angiography in the evaluation of the evolution of occult CNV.

\section{SIDE EFFECTS OF RADIOTHERAPY}

Various methods have been used including external beam radiation with dose regimens ranging from 8 to $24 \mathrm{~Gy}^{21-23} 2526$ Fraction schedules also varied widely ranging from 2 to $8 \mathrm{~Gy}^{21-23} 25263334$ Cumulated doses ranging from 10 to 15 Gy have been shown to be effective without causing any local or systemic side effects. ${ }^{21}{ }^{25}$ We used a total dose of $16 \mathrm{~Gy}$ in four sessions of $4 \mathrm{~Gy}$. According to previous reports ${ }^{26}$ no short term complications were observed during follow up that might preclude radiotherapy for occult subfoveal CNV.

In conclusion, external beam radiotherapy seems to have a beneficial effect on the evolution of occult subfoveal CNV. However, longer follow up is essential to evaluate final visual outcome and scar formation (as occult CNV have a slow natural history) to exclude any late onset side effect such as radiation induced retinopathy.

1 Kahn HA, Moorehead HB. Statistics on blindness in the model reporting area 1969-1970. United States Department of Health, Education and Welfare, Publ No (NIH) 73-427. Washington, DC: US Government Printing Office, 1973.

2 Vingerling JR, Dielemans I, Hofman A, et al. The prevalence of age related maculopathy in the Rotterdam study. Ophthalmology 1995;102:205-10.

3 Ferris FL III. Senile macular degeneration: a review of epidemiologic features. Am F Epidemiol 1983;118:132-51.

4 Young RW. Pathophysiology of age-related macular degeneration (review). Surv Ophthalmol 1987;31:291-306.

5 Macular Photocoagulation Study Group. Subfoveal neovascular lesions in age-related macular degeneration: guidelines for evaluation and treatment in the Macular Pho

6 Bressler NM, Frost LA, Bressler SB, et al. Natural course of poorly defined choroidal neovascularization associated with macular degeneration. Arch Ophthalmol 1988;106: 1537-42. 
7 Soubrane G, Coscas G, Francais C, et al. Occult subretinal new vessels in age-related macular degeneration: natural $649-57$.

8 Coscas G, Soubrane G. Photocoagulation des néovaisseaux sous-rétiniens dans la dégénéréscence maculaire sénile par laser à argon. Résultats de l'étude randomisée de 60 cas. Bull Mém Soc Fr Ophtalmol 1983;94:149-54.

9 Coscas G, Soubrane G, Ramahefasolo C, et al. Perifoveal laser treatment of subfoveal choroidal new vessels in age-related macular degeneration: results of a randomized clinical trial. Arch Ophthalmol 1991;109:1258-65.

10 Macular Photocoagulation Study Group. Persistent and recurrent neovascularization after krypton laser photocoagulation for neovascular lesions of age-related macular degeneration: results of a randomized clinical trial. Arch Ophthalmol 1990;108:825-31.

11 Macular Photocoagulation Study Group. Argon laser photocoagulation for neovascular maculopathy: five years photocoagulation for neovascular maculopathy: five years 1991;109:1109-14.

12 Macular Photocoagulation Study Group. Five-years follow-up of fellow eyes of patients with age-related macular degeneration and . Arch Ophthalmol 1993;111:1189-99.

13 Macular Photocoagulation Study Group. Laser photocoagulation of subfoveal neovascular lesions of age related macular degeneration; updated findings from two clinical trials. Arch Ophthalmol 1993;111:1200-9.

14 Macular Photocoagulation Study Group. Laser photocoagulation for juxtafoveal choroidal neovascularization: fiveyear results from randomized clinical trials. Arch Ophthal mol 1994;112:500-9.

15 Macular Photocoagulation Study Group. Visual outcome after laser photocoagulation for subfoveal choroidal neovascularization secondary to age-related macular degeneration: the influence of initial lesion size and initial visual acuity. Arch Ophthalmol 1994;112:480-8.

16 Soubrane G, Barreau E, Algan M, et al. Photocoagulation disséminée dans le cas de néovaisseaux sous-rétiniens disseminee dans le cas de neovaisseaux sous-retiniens occultes: resultat $1994 ; 4: 375-8$.

17 Bressler N, Maguire MG, Murphy PL, et al. Macular scatter ("grid") laser treatment of poorly demarcated subfoveal choroidal neovascularization in age-related macular degeneration. Arch Ophthalmol 1996;114:1456-64.

8 Macular Photocoagulation Study Group. Occult choroida neovascularization: influence on visual outcome in patients with age-related macular degeneration. Arch Ophthalmol 1996;114:400-12

19 Freund KB, Yannuzzi LA, Sorenson JA. Age-related macular degeneration and choroidal neovascularization. $A m \mathcal{f}$ Ophthalmol 1993;6:786-91.

20 Stevens TS, Bressler NM, Maguire MG, et al. Occult choroidal neovascularization in age-related macular degeneration. Arch Ophthalmol 1997;115:345-50.

21 Chakravarthy U, Houston RF, Archer DB. Treatment of age-related macular subfoveal neovascular membranes by age-related macular subfoveal neovascular membranes by

22 Bergink GJ, Deutman AF, Van den Broek JFCM, et al. Radiation therapy for subfoveal choroidal neovascular Radiation therapy for subfoveal choroidal neovascular
membranes in age-related macular degeneration. Graefes Arch Clin Exp Ophthalmol 1994;232:591-8.

23 Bergink GJ, Deutman AF, van den Broek JFCN, et al. Radiation therapy for age-related subfoveal choroidal neo- vascular membranes. A pilot study. Doc Ophthalmol 1995;90:67-74.

24 Hart PM, Archer DB, Chakravarthy U. Asymmetry of disciform scarring in bilateral disease when one eye is treated with radiotherapy. Br f Ophthalmol 1995;79:562-8.

25 Hart PM, Chakravarthy U, MacKenzie G, et al. Teletheraphy for subfoveal choroidal neovascularization: results of follow up in a non-randomized study. $\mathrm{Br} \mathcal{F}$ Ophthalmol 1996;80:1046-50.

26 Finger PT, Berson A, Sherr D, et al. Radiation therapy for subretinal neovascularization. Ophthalmology 1996;103: $878-99$.

27 Arnold J, Algan M, Soubrane G, et al. Indirect scatter laser photocoagulation for subfoveal choroidal neovascularization in age-related macular degeneration. Graefes Arch Clin Exp Ophthalmol 1997;235:208-16.

28 Pharmacological Therapy for Macular Degeneration Study Group. Interferon alfa-2 is ineffective for patients with choroidal neovascularization secondary to age-related macular degeneration. Arch Ophthalmol 1997;115:865-72.

29 Berger AS, Kaplan AG. Clinical experience with the surgical removal of subfoveal neovascular membranes. Ophthalmology 1992;99:969-76.

30 Thomas MA, Grand MG, William DF, et al. Surgical management of subfoveal choroidal neovascularization. Ophthalmology 1992;99:952-68.

31 Thomas MA, Dickinson JD, Melberg NS, et al. Visual results after surgical removal of subfoveal choroidal
neovascular membranes. Ophthalmology 1994;101:138496.

32 Ormerod LD, Puklin JE, Frank RN. Long term outcome after the surgical removal of advanced subfoveal neovascuar membranes in age-related macular degeneration. Ophthalmology 1994;101:1201-10.

33 Berson A, Finger PT, Sherr DL, et al. Radiotherapy for agerelated macular degeneration:preliminary results of a potentially new treatment. Int $\mathcal{F}$ Radiat Oncol Biol Phys 1996;36:861-5.

34 Schleicher U, Andreopoulos D, Wolf S, et al. Radiotherapy of macular degeneration: experience with two fractionation schemes. In: Wiegel T, Bornfeld N, Foerster MH, Hinkelbein W, eds. Radiotherapy of ocular disease. Basel: Karger, 1997;30:253-8.

35 Stalmans P, Leys A, Van Limbergen E. External beam radiotherapy (20 Gy, 2 Gy fractions) fails to control the growth of choroidal neovascularization in age-related macular degeneration: a review of 111 cases. Retina 1997; 17:481-92.

36 Chakravarthy U, Gardiner TA, Archer DB, et al. A light microscopic and autoradiographic study of non-irradiated and irradiated ocular wounds. Curr Eye Res 1989;8:33748

37 Jalkh AE, Nasrallah FP, Marinelli I, et al. Inactive subretinal neovascularization in age-related macular degeneration. Ophthalmology 1990;97:1614-19.

38 Guyer DR, Yannuzzi LA, Slakter JS, et al. Indocyanine green videoangiography of occult choroidal neovascularization. Ophthalmology 1994;101:1727-37.

39 Guyer DR, Yannuzzi LA, Slakter JS, et al. Classification of choroidal neovascularization by digital indocyanine videoangiography. Ophthalmology 1996:103:2054-60.

40 Yannuzzi L, Slakter J, Sorensen J, et al. Digital indocyanine green videoangiography and choroidal neovascularization. Retina 1992;12:191-223. 